\title{
PROPOSED VISUAL WIKI SYSTEM FOR GATHERING KNOWLEDGE ABOUT DISCRETE EVENT SYSTEMS
}

\author{
Peter Dungan \\ Cathal Heavey \\ University of Limerick \\ Limerick, IRELAND
}

\begin{abstract}
The first phase of the conceptual modeling process is the acquisition of knowledge about the real world system. One issue in this phase is the need for clear communication, between the modeler, and experts on the system being examined. These domain experts may not be versed in modeling techniques or languages. Another issue is the potential benefit offered by the recording of the gathered knowledge, in a way that facilitates its reuse outside of the modeling project itself. Existing approaches to the construction of a system description have different strengths and weaknesses. Therefore a combination of different model types, in an integrated manner, could be most effective. Visual wiki software is proposed to facilitate this. Wikis are proven as a platform for incrementally growing shared knowledge bases. They are generally text-based; a wiki allowing editing of graphics as well as text would be preferable for system and process knowledge.
\end{abstract}

\section{INTRODUCTION}

Models of discrete event systems need to contain enough detail for an accurate simulation of the system to be created. They also should be understandable without special training. In practice, these two requirements conflict to an extent; it is often difficult to satisfy both.

The need for the model to be understandable is particularly important in the initial, knowledge gathering phase. In this phase, a description of the real system is constructed, which is then abstracted into a description of the simulation (see Figure 1). This phase requires communication between the modeler and experts on the system being modeled, who are not necessarily fluent in modeling languages or methodologies. For them to be able to verify the correctness of a model, they must be able to interpret the model. It is important that they are able to verify the model is correct at the earliest stage possible. It is far less costly to identify errors in a model early on; in particular it is less costly to identify errors prior to the coding and use of a model, than afterwards.

A second reason it is highly preferable that the model is described in a way that is easy to understand, is that it would be preferable to be able to reuse knowledge gathered about the system. The task of gathering knowledge is often non-trivial, and requires a significant amount of time and effort. It would be useful to store this knowledge in a way that facilitates its further development and reuse. Neither further development nor reuse is likely when the only artifact that is understandable to non-experts is the simulation model itself. It may be beneficial to apply existing enterprise knowledge management techniques. 


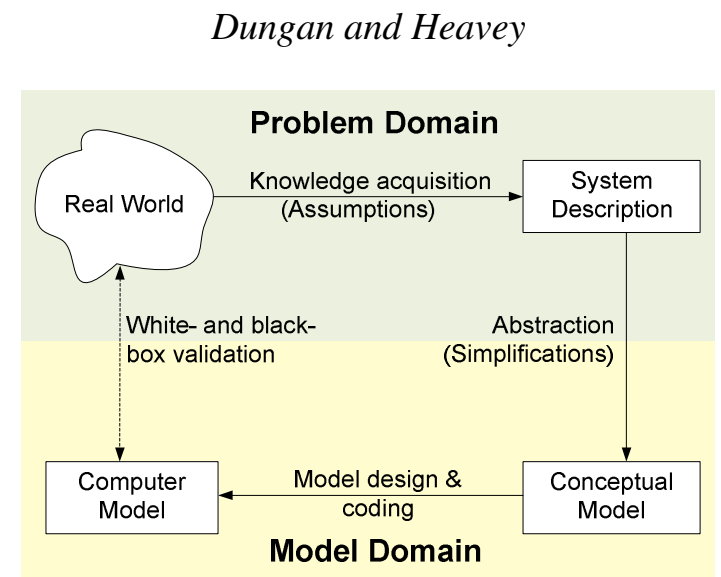

Figure 1: Artifacts of Conceptual Modeling; adapted from (Kotiadis and Robinson 2008).

An established tool in knowledge management is the wiki. Wikis are widely used in both enterprises and Websites. They allow knowledge to be distributed easily, and for it to be updated incrementally without a significant learning curve. A wiki is an excellent medium to store knowledge in, from the point of view of communication and of development of the knowledge. However the utility of a traditional wiki in describing discrete event systems is limited. Firstly, it does not facilitate the editing of diagrams and images, which are an important part of many methods of describing discrete event systems. Secondly, it does not provide a means of modeling the system at a logical level.

However, technology has advanced since the invention of the wiki, and it is now possible to address these issues. Much faster internet access speeds, and the development of rich internet application frameworks means that a wiki which allows a high level of graphic and diagram editing, as well as text, is now feasible. The emergence of Web 3 has led to the development of the semantic wiki, which has an underlying model of the knowledge contained in its pages (Boulos 2009).

The proposed wiki system needs to be able to represent system knowledge in a manner that can describe complex systems accurately, and it needs to be able to communicate this knowledge to non-expert users. The wiki addresses these issues by facilitating the combination of different diagram types. Relatively unrestricted illustrative content would be used to aid communication

Combining this with formal logical definitions of the content, might enable the integration of different modeling standards within the same system. A system which provides the ability to define the logical and graphical components of a model at a primitive level would be of considerable utility. It would enable the definition of different forms of graphical models in the same integrated environment.

The next section is a brief review of the literature on the pre-coding phase of simulation projects, and on wikis. Following that is a description of the proposed system, and a prototype is presented. Finally, the conclusions are presented.

\section{CONCEPTUAL MODELING FOR DISCRETE EVENT SIMULATION}

Conceptual modeling is an important phase of a discrete event simulation project. It is recommended that $40 \%$ of project time is allocated to this phase (Shannon 1981; Ryan and Heavey 2006). The impact of conceptual modeling activities on other phases (implementation and validation) of the project is significant. It provides a blueprint of what to model in the implementation phase. Errors in the conceptual model result in errors in the model implementation, and ultimately in inaccurate results.

As mentioned, this is a problematic area. Existing approaches generally fail to satisfy both the requirements of conveying sufficient detail, and being understandable to non-experts. There is a lack of research and support in this area (Benjamin, Delen et al. 2000; Ryan and Heavey 2006). In practice, a simulation designer often skips directly into the code itself, leaving no system or design documentation at all (Benjamin, Delen et al. 2000; Arbez and G. Birta 2007). 


\section{Dungan and Heavey}

Another issue is the process of gathering necessary knowledge. It is common for a modeller to find that the information required to build a model is not available, even in instances where the organisation is awash with data (Pidd 2009). In larger simulation projects, the gathering of data and development of designs is nontrivial. It is likely that the knowledge required to build the model will need to be gleaned from numerous different sources. For example, process design involves many disciplines and is highly collaborative. In this area, it is estimated that engineers spend $60 \%$ of their time searching for data from disparate sources, collating and transcribing the data, and filing the results (Britt, Chen et al. 2004).

Although they are generally treated separately in the literature, there are many links between knowledge management and simulation development (Hlupic, Verbraeck and de Vreede 2002). Every stage of a simulation project could be mapped to a knowledge management activity, where a mutually beneficial relationship could be established (Hlupic, Verbraeck and de Vreede 2002).

\section{WIKIS}

A wiki is a Website where users can edit and create content (Hasan, Meloche et al. 2007; Hester and Scott 2008), and easily create links between topics (Hester and Scott 2008). Leuf and Cunningham (2001) define a wiki as "a hypertext system for storing and modifying information - a database where each page is easily editable by any user within a forms-capable Web browser client"(Blasche and Stein 2008).

\subsection{General Characteristics}

Typically, a history of changes made is kept (Huner and Otto 2009). Most wiki software has searching and indexing capabilities (Blasche and Stein 2008).

Wikis are simple to use. It is possible to edit content pages directly by clicking a link or button. The actual edit is generally done using simple markup, though some systems provide WYSIWYG ("What you see is what you get") editors (Fichter 2005). Most wiki implementations have rules or guidelines about style for content. In fact the philosophy of how content should be edited can be considered to be part of the definition of a wiki (Louridas 2006). In order to maintain the desired style in a wiki, members of the user community volunteer to revise content, add links, and remove vandalism (Fichter 2005).

There are some common features for navigation in wikis. One defining feature of the wiki is the easy creation of hyperlinks in the page content (Huner and Otto 2009). Generally words in a paragraph of text are marked as links which open new pages giving more detail on the marked terms. The standard menus and submenus, that provide navigation for many Websites, often aren't available in a wiki. Instead, a search feature should be ubiquitous, and 'Road Map' and 'Starting Point' pages are often defined. Additionally, some wikis allow pages to be tagged or categorized. It is also usually possible to see a list of backlinks (i.e.: a list of the pages that link to the current page) by clicking the page title (Fichter 2005).

\subsection{Wikis in Enterprise}

Wikis are used a great deal in enterprise. Bughin (2008) describes a survey of over 2,800 executives on the adoption of collaborative Web 2.0 technologies. The survey was worldwide and across different industries. $13 \%$ of respondents stated that their company had invested in the use of wikis. They concluded that recognition for contribution was important for encouraging participation in tools such as wikis. They also concluded that the rate of adoption of such technologies is on par with, or faster, than other IT-based technologies.

Wikis can facilitate collaboration between large numbers of users in the creation of a library of knowledge. Knowledge can be created by individuals or by collaborative groups (Hasan, Meloche et al. 2007). Since the success of Wikipedia, wiki software has become a broadly discussed alternative for corporate metadata management (Huner and Otto 2009). Research has suggested that a level of ongoing interest from management in an enterprise wiki project is necessary for its success (Grudin 2006; Hasan, Meloche et al. 2007). 


\section{Dungan and Heavey}

Danis and Singer (2008) designed and deployed a wiki-based application supporting yearly planning work by members of a globally distributed, 900-member research organisation (the goal to enable greater transparency and provide a basis for broader collaboration). As a result of their study, they concluded that the wiki in the enterprise has special characteristics.

They found there is a need to restrict access in wikis in enterprise. Power relationships and competition between stakeholders created a need to read access in the ResearchWiki, unlike a typical public wiki.

They perceived that users were reluctant to modify each other's content, except for in special circumstances, such as members of the same project.

A survey of 168 corporate wiki users was conducted by Majchrzak, Wagner and Yates (2006). They identifed different types of users in a wiki, and categorised them as adders and synthesisers. Synthesisers provided contributions involving integration, reorganization and rewriting whole paragraphs could be clustered together. Adders added content and created topics and pages. They concluded that synthesisers are more interested in impact, while adders are more interested in accomplishing their immediate work responsibilities. They suggested seeking out impact-oriented individuals and utilitarian-oriented individuals to ensure that a wiki has both synthesisers and adders (Majchrzak, Wagner and Yates 2006).

They concluded that corporate wikis are sustainable. Three main types of benefits of wiki use were identified by the users: enhanced reputation, work made easier, and helping the organization to improve its processes. They concluded that not all corporate wikis generate these benefits however. The benefits were more likely to be perceived when tasks require novel solutions, and when other users are believed to provide credible information. They also found that building of reputation was more likely to benefit users with a high level of expertise, and that work benefits were more likely associated with task interdependence, availability of alternative communication channels, and membership in the core group (Majchrzak, Wagner and Yates 2006).

Research into failed corporate wiki implentations has identified issues that mitigate against the easy uptake of Wikis in corporations (Hasan, Meloche et al. 2007):

- The informal network approach that is currently favored in a Wiki, implies loss of central management control of corporate knowledge and changes to organizational structure and culture

- The Wiki is described as 'social software', implying that there are social factors that must undergo some changes before the Wiki will be accepted to improve the organization's knowledge management

- Legal issues concerning rights to intellectual property and possible libelous material see a Wiki as a risky endeavor.

\subsection{Visual Wikis}

Wiki technology is designed for manipulating text, not images (Viegas 2007). As a result, collaborating around pictures turns out to be more difficult than collaborating around text (Viegas 2007). They are typically attached files in wiki pages, and exist separately to the wiki site (Viegas 2007). Wikis were invented before it was feasible to handle graphics in them in a more sophisticated manner.

There are recent advances in internet technology which facilitate the development of new forms of online knowledge management tools. Tools for constructing rich internet applications have emerged which allow a far higher level of interactivity in Web applications. These advances make it possible to handle images as part of the wiki content itself, rather than attached separate files. Some wiki-style tools based on these technologies have been proposed in the literature. Also some wiki-style tools have been released in the industry which are based on these emergent technologies. Hirsch, Hosking et al. (2009) put forward various visual wiki prototypes. ThinkBase and VKBE are two prototypes which present a navigable visualization of the structure of semantic wikis. User feedback on these indicated that such a visual interface was very well received. 


\section{Dungan and Heavey}

Another prototype put forward by Hirsch, Hosking et al. (2009) is ProcessMapper. This allows processes to be defined in BPMN, which is mapped to a text wiki. Editing the visual display results in changes to the wiki page (although the reverse is not true). This is intended to be used as a process documentation repository.

\section{VISUAL WIKI FOR GATHERING KNOWLEDGE ABOUT DISCRETE EVENT SYSTEMS}

Systems knowledge is apt to be described in a visual manner. Bringing interactive graphical functionality into a wiki makes it more suitable for describing systems.

A wiki offers benefits as a means of storing knowledge. Wikis foster collaboration, thanks to their ease of use and of access, and full traceability and versioning of content. They engage users, because content is structured and accessed in a way that mirrors human thought processes (Ullman and Kay 2007). This feature makes them easy to navigate, and useful as tools for teaching and learning.

These particular benefits suggest a visual wiki would be of significant use when knowledge is being gathered about a discrete event system in a simulation project: Significant effort and resources are often spent in communicating with domain personnel about the system being modeled results (Britt, Chen et al. 2004). Furthermore, the knowledge gathered tends not to be stored in a manner fit for reuse (Arbez and G. Birta 2007; Pidd 2009). A visual wiki could address both these issues. Another aspect of the visual wiki described here is that users are not restricted to predefined modeling elements, but can define their own, or use a free-form approach. Generally people will naturally use free-form diagrams to describe systems, so this would be likely to encourage contribution, particularly from novice users. This could be beneficial in the context of gathering knowledge for discrete event systems, where domain experts would not necessarily be versed in modeling standards or techniques. This feature of the software presents possible issues however, since there would be no standardization in the model, and validation and verification of content could be made more complex.

\subsection{Features of the Proposed Visual Wiki}

The proposed software is a visual wiki. Hirsch's definition of this is a visualization which maps to a textbased semantic wiki page. This does not fit the visual wiki presented here. The visual wiki described here is an application of the wiki concept to graphical content. i.e.: Graphical content can be manipulated in the same easy way that textual content can be manipulated in a traditional wiki. The software allows users to define model elements, compile these into custom templates (i.e.: collections of related diagram or model constructs) and to construct models from these. The Visual Wiki shares a number of characteristics with a traditional wiki. Full versioning and traceability of all content is maintained, and it runs in a standard internet browser, without any need for local installation. Users can navigate through the content in a similar manner to traditional wikis, via decomposition of model elements, and links to related content. The content can be searched. Access and edit rights to models and model components can be defined, and applied to individual users or to user groups (roles). In this way, all content can be shared or kept private. Allowing diagram elements to be defined in the wiki allows different standards to be combined and annotated. This could be useful in communicating knowledge in the system across a varied audience. Also narratives or annotations could be added to aid interpretation of less intuitive standards. Model elements are drawn directly inside the wiki. They are rendered as anti aliased vector graphics. Data properties may be defined, of different types (numeric or text). These properties can be queried, and rules and relations can be defined to trigger visual changes in objects based on these property values, such as position, size, line and fill colour, opacity. This has a wide range of possible uses, from highlighting areas in the system of interest to generating graphs. It would be possible to integrate the software with external data sources, via its API. 


\section{Dungan and Heavey}

\subsection{Implementation}

An illustration of the architecture of the visual wiki is shown in Figure 2. Silverlight applications run in a sandbox on the client, which are downloaded and cached on the client. Models are navigated to via post parameters in the URLs so they can be bookmarked, and linked to directly. The Silverlight application can be embedded in any Web page.

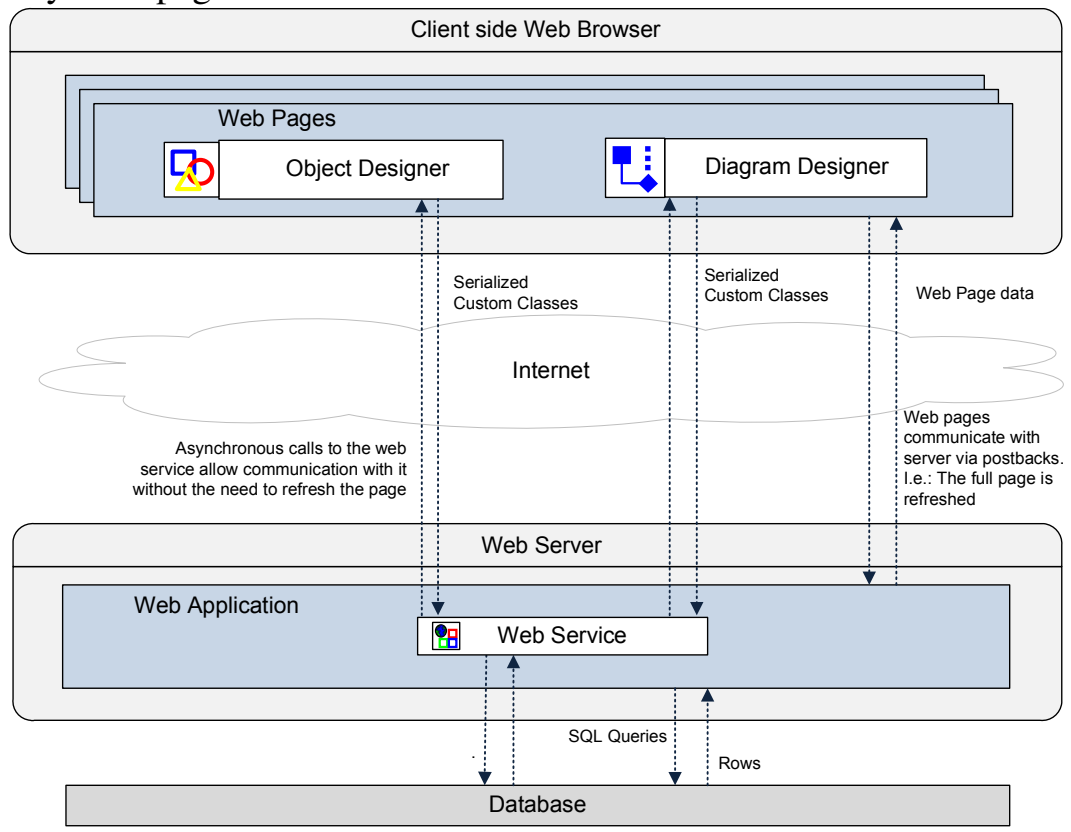

Figure 2: Overview of the prototype architecture

\subsection{Prototype}

To test and validate the concept of a Visual Wiki in the management of system knowledge, prototype software has been developed. The current version of the prototype allows the building of graphical object templates and has the features described in section 4.1 implemented, i.e., versioning and traceability and access/edit rights. An example template is shown in Figure 3.

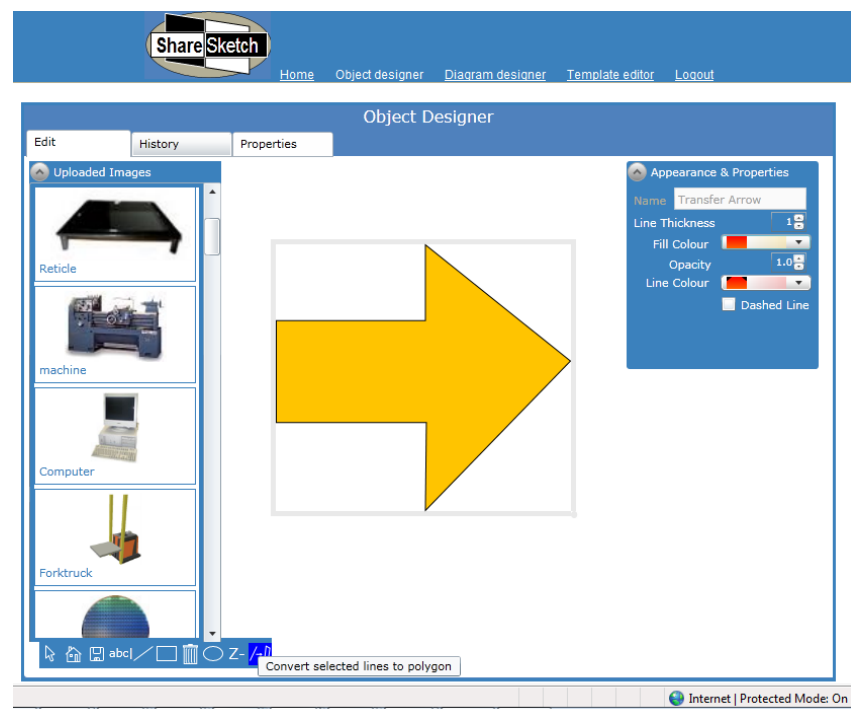

Figure 3: Interface for defining graphical objects in the prototype 


\section{Dungan and Heavey}

An example of how this template can be used to describe a system is shown in Figure 4. The next phase of the work is to use the prototype software to explore further the concept of using a modeling environment that integrates several modeling paradigms embedded within a wiki framework. This will be accomplished using a case based approach. Finally, it is important to note that the current prototype software only depicts visually a modeling element, it does not capture logical aspects.

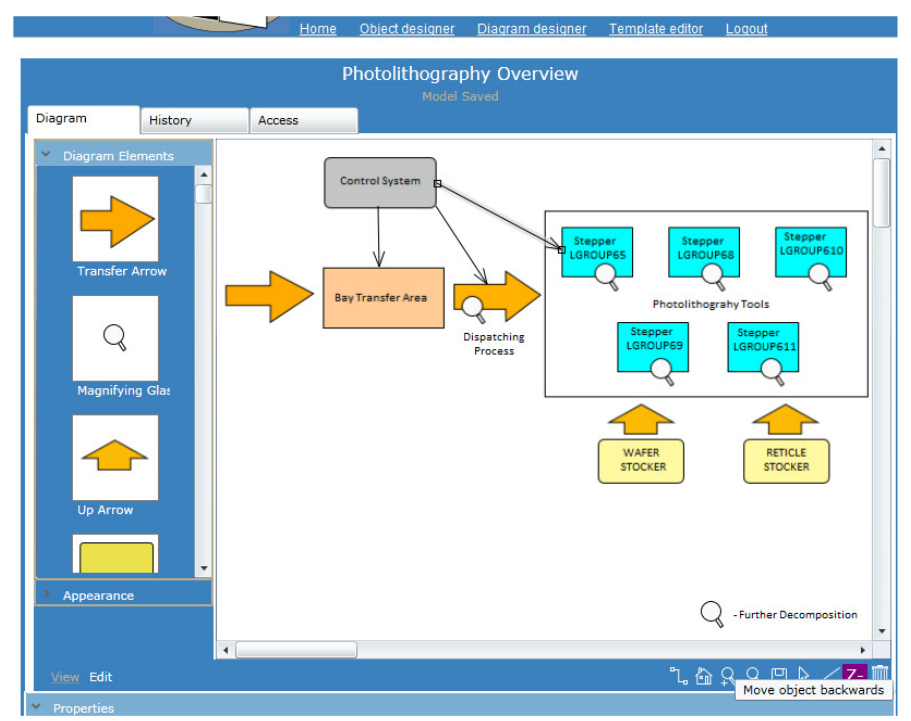

Figure 4: Interface for using a template to define a system

\section{CONCLUSIONS}

This paper proposes the use of the wiki concept for capturing and managing process knowledge, in a similar manner that enterprise wikis are currently widely used to capture corporate knowledge. As current wiki technology only has very limited support for graphical modeling, prototype software has been developed to explore further the concept. The current version of the prototype software allows model templates to be developed and also has integrated within the software wiki concepts, such as, versioning and traceability and access/edit rights and the tool is fully web enabled. Future planned work is to use the prototype software to evaluate using case studies the Visual Wiki concept.

\section{REFERENCES}

Arbez, G. and L. G. Birta. 2007. ABCmod: A Conceptual Modelling Framework for Discrete Event Dynamic Systems. Summer Computer Simulation Conference, Society for Computer Simulation International: 987-995.

Benjamin, P., Delen D., Mayer, R. and T. O'Brien. 2000. A model-based approach for component simulation development. In Proceedings of the 2000 Winter Simulation Conference, eds. J. A. Joines, R. R. Barton, K. Kang, and P. A. Fishwick. Piscataway, New Jersey: Institute of Electrical and Electronics Engineers, Inc.

Blasche, S. and K. Stein. 2008. "Methods and Measures for the Analysis of Corporate Wikis: A Case Study." Paper presented at the annual meeting of the International Communication Association, Montreal, Quebec, Canada, May 21, 2008

Boulos, M. N. K. 2009. "Semantic Wikis: A Comprehensible Introduction with Examples from the Health Sciences." Journal of Emerging Technologies in Web Intelligence 1(1): 94.

Britt, H., C. C. Chen, V. Mahalec and A. McBrien. 2004. Modeling and simulation in 2004: An industrial perspective. Proc. of FOCAPD: 11-16. 


\section{Dungan and Heavey}

Bughin, J. 2008. "The rise of enterprise 2.0." Journal of Direct, Data and Digital Marketing Practice 9(3): 251-259.

Danis, C. and D. Singer. 2008. "A Wiki Instance in the Enterprise: Opportunities, Concerns and Reality." Proceedings of CSCW'08.

Fichter, D. 2005. "Intranets, wikis, blikis, and collaborative working." ONLINE-WESTON THEN WILTON- 29(5): 47.

Grudin, J. 2006. "Enterprise Knowledge Management and Emerging Technologies." Proceedings of the 39th Hawaii International Conference on System Sciences.

Hasan, H., J. Meloche, Pfaff, C.C. and D. Willis. 2007. Beyond ubiquity: Co-creating corporate knowledge with a wiki. Mobile Ubiquitous Computing, Systems, Services and Technologies, 2007. UBICOMM'07. International Conference on: 35-40.

Hester, A. J. and J. E. Scott 2008. A Conceptual Model of Wiki Technology Diffusion. Proceedings of the 41st Hawaii International Conference on System Sciences.

Hirsch, C., J. Hosking, Grundy, J., Chaffe, T., MacDonald, D. and Y. Halytskyy. 2009. The Visual Wiki: A New Metaphor for Knowledge Access and Management. Proceedings of the 42nd Hawaii International Conference on System Sciences.

Hlupic, V., A. Verbraeck and G. J. de Vreede. 2002. SIMULATION AND KNOWLEDGE MANAGEMENT: SEPARATED BUT INSEPARABLE? 14th European Simulation Symposium, 14th European Simulation Symposium.

Huner, K. M. and B. Otto 2009. "The Effect of Using a Semantic Wiki for Metadata Management: A Controlled Experiment." Proceedings of the 42nd International Conference on System Sciences.

Kotiadis, K. and S. Robinson 2008. Conceptual modelling: knowledge acquisition and model abstraction. In Proceedings of the 2008 Winter Simulation Conference, eds. S. J. Mason, R. R. Hill, L. Mönch, O. Rose, T. Jefferson, J. W. Fowler. Piscataway, New Jersey: Institute of Electrical and Electronics Engineers, Inc.

Leuf, B. and W. Cunningham 2001. The Wiki way: quick collaboration on the Web, Addison-Wesley Longman Publishing Co., Inc. Boston, MA, USA.

Louridas, P. 2006. "Using Wikis in Software Development." IEEE Software, March/April.

Majchrzak, A., C. Wagner and D. Yates. 2006. Corporate Wiki Users: Results of a Survey. In Proc. ACM Wikisym, 2006. Pages: 99-104.

Pidd, M. 2009. Why Modelling Still Matters in Discrete Simulation. 2009 INFORMS Simulation Society Research Workshop.

Ryan, J. and C. Heavey 2006. "Process Modelling for Simulation." Computers in Industry 57(5): 437-450.

Shannon, R. E. 1981. "Tests for the verification and validation of computer simulation models.". In Proceedings of the 1981 Winter Simulation Conference. 573-577.

Ullman, A. J. and J. Kay 2007. WikiNavMap: a visualisation to supplement team-based wikis, ACM.

Viegas, F. B. 2007. The Visual Side of Wikipedia. Proceedings of the 40th Hawaii International Conference on System Sciences.

\section{AUTHOR BIOGRAPHIES}

PETER DUNGAN is currently pursuing the Ph.D. degree in the Enterprise Research Centre at the University of Limerick, Ireland. His bachelor's degree is in Applied Computing from Waterford Institute of Technology. He has industry experience as a software developer and as an analyst. His main research interest is in the development of collaborative knowledge management software. He can be reached via email at $<$ peter. dungan@ul. ie $>$.

DR. CATHAL HEAVEY is the head of the Department of Manufacturing and Operations Engineering at the University of Limerick. $\mathrm{He}$ is an Industrial Engineering graduate of the National University of Ireland (University College Galway) and holds a M.Eng.Sc. and Ph.D. from the same 
University. He has published in the areas of queuing and simulation modelling. Research interests are: Simulation Modelling of Discrete Event Systems; Modelling and Analysis of Supply Chains and Manufacturing Systems; Process modeling. His email address is <cathal. heaveyeul.ies. 